\title{
Revista Historia de la Educación Latinoamericana, líder en la investigación histórico-educativa 1998-2013
}

\author{
Diana Soto Arango ${ }^{1}$ \\ Universidad Pedagógica y Tecnológica de Colombia - Tunja (Colombia) \\ Grupo de investigación HISULA \\ dianaelvirasoto@gmail.com \\ Sandra Liliana Bernal Villate ${ }^{2}$ \\ Universidad Pedagógica y Tecnológica de Colombia - Tunja (Colombia) \\ Grupo de investigación HISULA \\ saralili55@hotmail.com
}

Recepción: 17/09/2012

Evaluación: 04/10/2012

Aceptación: 15/11/2012

Artículo de Reflexión

DOI: http:/ / dx.doi.org / 10.9757 / Rhela.20.02

\section{RESUMEN}

El objeto de este trabajo es realizar un balance y prospectiva de la Revista Historia de la Educación Latinoamericana (RHELA) desde el trabajo editorial durante sus 15 años de existencia, destacando las condiciones de producción, distribución, recepción y consulta, desde un análisis temático en la dimensión latinoamericana de esta publicación. En su calidad de pionera y única en su género, RHELA es una publicación científica y medio de expresión de la Sociedad de Historia de la Educación Latinoamericana (SHELA), del Doctorado en Ciencias de la Educación y de los grupos de investigación Historia y Prospectiva de la Universidad Latinoamericana (HISULA) y La Ilustración en América Colonial (ILAC), adscritos a la Universidad Pedagógica y Tecnológica de Colombia. La Revista está dirigida a investigadores en el campo de la historia de la Educación Latinoamericana, a sociólogos, edu- cadores, politólogos, y profesionales vinculados con el ámbito de las ciencias sociales, humanas y la educación. Esta Revista ha logrado mantenerse gracias al apoyo académico de los investigadores. Ha presentado impacto académico-investigativo, por ser un medio de consulta referenciado. Se cataloga en Índices internacionales y se encuentra clasificada en Publindex Colciencias en categoría " $\mathrm{B}$ ", se edita en físico, y en medio electrónico en la web de la UPTC y de RUDECOLOMBIA. A la fecha, este número 20 se ha editado con 12 artículos de 14 autores de 5 países, entre los que se destacan Argentina, Colombia, Costa Rica, Cuba y México.

Palabras clave: Revista Historia de la Educación Latinoamericana, educación, historia, SHELA, RUDECOLOMBIA.

1 Profesora titular de la Universidad Pedagógica y Tecnológica de Colombia. Con doctorado y posdoctorado del CSIC. Estudió en la UNED y trabajo en la UNED de Guinea Ecuatorial. Organizó en 1996 el Doctorado en Ciencias de la Educación de RUDECOLOMBIA, del que fue la primera directora. Organizó en 1992 la Sociedad de Historia de la Educación Latinoamericana y es la Directora de los Grupos de Investigación: Historia y Prospectiva de la Universidad Latinoamericana (HISULA) y la Ilustración en América Colonial (ILAC), visibles en Colciencias. Actualmente, es la coordinadora Sala Ciencias Humanas, Ciencias Sociales y Educación, CONACES. Directora de la Revista Historia de la Educación Latinoamericana-RHELA.

2 Candidata a Doctor en Historia de América por la Universidad Pablo de Olavide de Sevilla (España), Magister en Historia de América Latina de la Universidad Pablo de Olavide de Sevilla (España), licenciada en Ciencias Sociales de la Universidad Pedagógica y Tecnológica de Colombia. Actualmente se desempeña como catedrática externa de la Escuela de Sociales de la UPTC y como asistente de publicaciones e internacionalización del Doctorado en Ciencias de la Educación CADE-UPTC. 
Journal History of Latin American Education, leader in the historical-educational research eading educational research 1998-2013

\section{ABSTRACT}

The purpose of this paper is to take a stock and a foresight of the Journal History of Latin American Education, from the editorial work during its 15 years of existence, it has highlighted the conditions of production, distribution, reception and searching, from a thematic analysis in the Latin American dimension of this publication. In its quality of pioneering and the one in its genre, RHELA is a scientific publication and it is a way of expression of the Society of History of the Latin-American Education - SHELA of the Doctorate in Sciences of the Education and of the research teams History and Futurology of the Latin-American University - HISULA and The Enlightenment in Colonial America ILAC. The magazine is directed to researchers in the field of the history of the Latin-American Education, to sociologists, educators, political experts, and professionals involved in the field of social sciences, humanities and education. It is managed to hold support to the academic researchers. It has presented academic research impact by being a means of consultation referenced. It catalogs in international Índex is Ranked in Publindex - Colciencias in category ' $\mathrm{B}$ ' and is published in physical and electronic means RUDECOLOMBIA website.

Key words: History of Latin American Education Journal, education, history, SHELA, RUDECOLOMBIA.
Revista História da Educação Latino-Americana, líder na pesquisa histórico-educativa 1998-2013

\section{RESUMO}

O objeto deste trabalho é realizar o balanço e a prospectiva da Revista História da Educação Latinoamericana, considerando o trabalho editorial durante seus 15 anos de existência, destacando as condições de produção, distribuição, recepção e consulta, a partir de uma análise temática na dimensão latino-americana desta publicação. Em sua qualidade de pioneira e única em seu gênero, RHELA é uma publicação científica e meio de expressão da Sociedade de História da Educação Latino-americana - SHELA, do doutorado em Ciências da Educação e dos grupos de pesquisa História e Prospectiva da Universidade Latinoamericana - HISULA e A Ilustração na América Colonial - ILAC. Atribuído à Universidade Pedagógica e Tecnológica da Colômbia A revista está dirigida a pesquisadores no campo da História da Educação Latino-americana, a sociólogos, educadores, politólogos e profissionais vinculados com o âmbito das Ciências Sociais, Humanas e Educação. Alcançou o êxito de se manter pelo apoio acadêmico de seus colaboradores. Apresentou impacto acadêmico-investigativo por ser um meio de consulta referenciado. Está catalogada em Índex Internacional e se encontra Classificada em Publindex - Colciencias na categoria "B" e se edita em texto impresso e meio eletrônico na web de lá UPTC y RUDECOLOMBIA. Até esta data, incluindo-se este número 20 , foram côn 12 artigos de 14 pesquisadores pertencentes a 5 países entre os quais se destacam, Argentina, Colombia, Costa Rica, Cuba y México.

Palavras-chave: Revista História da Educação Latino-americana, educação, história, SHELA, RUDECOLOMBIA.

\section{INTRODUCCIÓN}

El objeto de este trabajo es presentar un análisis histórico de la Revista Historia de la Educación Latinoamericana, con motivo de los quince años de existencia y publicación permanente. Se presentará el perfil histórico desde el contexto latinoamericano y como proyecto académico-investigativo de publicación 
de la generación de los historiadores de la educación en Latinoamérica de finales del Siglo XX, que se encontraron y se fusionaron en dos proyectos académicos: La Sociedad de Historia de la Educación Latinoamericana y el Doctorado en Ciencias de la Educación de RUDECOLOMBIA.

La Revista es de carácter científico, tuvo una edición periódica anual hasta el 2007. Desde el año 2008 se ha editado en la modalidad bianual y para el 2012 se presentó en versión online en español e inglés. La publicación se dirige a investigadores en el campo de la historia de la Educación Latinoamericana, a sociólogos, educadores, politólogos, y profesionales vinculados con el ámbito de las ciencias sociales, humanas y la educación. RHELA ha logrado mantenerse gracias al apoyo académico de los grupos y proyectos de investigación vinculados a la Sociedad de Historia de la Educación Latinoamericana. ${ }^{3}$ Está registrada en el Sistema Nacional de Indexación y Homologación de Revistas Especializadas de CT+I Publindex Colciencias en categoría B; Fuente Académica (EBSCO - México); en Citas Latinoamericanas en Ciencias Sociales y Humanidades (CLASE); en el Instituto de Investigaciones sobre la Universidad y la Educación (IISUE); en los sistemas de hemeroteca, de acceso libre de la Red de Revistas Científicas de América Latina y El Caribe, España y Portugal (REDALYC) y en el Portal de difusión de la producción científica hispana (DIALNET), en el Directorio de Open ACCES (DOAJ).

Asimismo, se localiza en versión online ISSN 2256-5248 en la plataforma de uso editorial open Journal systems: http://www.rhela.rudecolombia.edu. co, http: / / virtual.uptc.edu.co/revistas/index.php/historia_educacion_ latinoamerica y http: / / www.shela-hedu.org.

Como método empleado para el análisis de la Revista, se estableció una visión teórica desde la historia de la educación, y de manera interdisciplinaria se procedió a construir un modelo para la investigación de esta producción intelectual del texto escrito, como espacio de estudio de redes de intelectuales, y así poder dar respuesta a los interrogantes que se establecen sobre este tipo de publicaciones que tienen una historia con una prospectiva de construcción de comunidades académicas. ${ }^{4}$ Por lo tanto, la pregunta para el análisis se centró en ¿RHELA es un espacio de visibilización, de investigacio-

3 Entre estos grupos de investigación se destaca la participación del grupo Historia y Prospectiva de la Universidad Latinoamericana (HISULA) y la Ilustración en América Colonial (ILAC), adscritos a la Universidad Pedagógica y Tecnológica de Colombia.

4 Joseph Fontana, Historia. Análisis del pasado y proyecto social (Barcelona: Crítica. Libro básico, 1982). 
nes, de un grupo de intelectuales con objetivos comunes y tiene la capacidad de liderar e impactar en el contexto internacional a la comunidad académica de historiadores de la educación y trascender en políticas públicas latinoamericanas?

En la metodología se establecieron como procedimiento de análisis varios indicadores. El primero es la historia de la organización de la Revista como producto de una idea de la Sociedad de Historia de la Educación Latinoamericana, identificando al grupo organizador y la comparación con otras revistas educativas en Colombia. Segundo, la red de intelectuales en la que se analiza la evolución del grupo editorial y de colaboradores de esta (director, co-directores, comités, colaboradores revisores, corresponsales, lectores, traductores), incluyendo a quienes han escrito y sobre qué temáticas lo han realizado. El tercer aspecto se establece en el contenido, en primera instancia, el análisis de editoriales, en el índice las secciones en su evolución, temas, ilustraciones, reseñas y páginas. Por otra parte, se analizarán los educadores que se han visibilizado, estudiando su procedencia (país), el impacto reformador de este y la corriente educativa-pedagógica que adaptó o creó el citado educador. Por ser una misma línea de pensamiento, se correlacionará con los educadores, a los que se les ha dedicado la caratula del Boletín Historia de la Educación Latinoamericano. De la misma manera, en el contenido se establecerán las temáticas que se han desarrollado con las líneas y proyectos de investigación. Como cuarto indicador, se describirá la Revista en los aspectos técnicos de: formato, diseño (interno, carátula), impresión en papel, encuadernación, páginas, lugar, difusión en bibliotecas, ferias, ventas, webs, reseñas, citaciones, repositorios, indexación. Quinto, internacionalización e impacto de la Revista y el grupo que la conforma, el papel que esta ha desempeñado para cohesionar la construcción de la comunidad académica. Sexto, dificultades, logros, y prospectiva. Séptimo, conclusiones.

Las fuentes de información fundamentalmente se sustentan en los números de la Revista, revisión bibliográfica y conceptos por entrevistas de sus miembros. Se concluye que RHELA ha impulsado un proyecto científicoacadémico donde se unen, bajo un mismo objetivo, una Sociedad académica y un programa de formación doctoral. 


\section{Antecedentes. La generación pionera de los proyectos científico- educativo en la Historia de la Educación Latinoamericana}

En septiembre de 1992 SHELA coordinó el I Congreso Iberoamericano de Historiadores de la Educación Latinoamericana en Bogotá, con la Red de Historiadores de la Educación Latinoamericana, que se venía organizando desde $1989^{5}$ y en el cual participaron representantes de 22 países Iberoamericanos. En las conclusiones de este evento se indicó que no existía en ese momento un Doctorado en Historia de la Educación en Latinoamérica y que era necesario organizar una sociedad académica que impulsara investigaciones en esta área del conocimiento. Los objetivos, del grupo que se impulsó en este evento académico, se fueron cristalizando a través del tiempo. La Sociedad de Historia de la Educación Latinoamericana se organizó en septiembre del año 1994 con motivo del II encuentro de la Red de Historiadores de la Educación Latinoamericana en Campinas, Brasil. En el año 1996 se propuso el proyecto de un Doctorado en Historia de la Educación Latinoamericana, el cual fue asumido por universidades colombianas y en junio de 1998 se cristalizó este proyecto con la aprobación del programa doctoral por parte del Ministerio de Educación Nacional de Colombia. ${ }^{6}$

Precisamente, es en este año cuando la comunidad académica de la Sociedad de Historia de la Educación Latinoamericana, que era la misma que impulsaba el Doctorado en Ciencias de la Educación con el área de Historia de la Educación Latinoamericana, visualizó que era imprescindible tener un órgano de expresión para dar a conocer los avances de investigación de esta comunidad académica. De esta manera, se fusionó en un solo proyecto académico-editorial SHELA con el doctorado de RUDECOLOMBIA. ${ }^{7}$

La Revista Historia de la Educación Latinoamericana edita su primer número el 8 de mayo de 1998 con el apoyo económico de los miembros de Sociedad Historia de la Educación Latinoamericana y las universidades de RUDECOLOMBIA. Esta primera edición aparece bajo la dirección de la Dra. Diana Soto Arango, directora del Doctorado en Ciencias de la Educación y coordi-

5 En el año 1989 se organizó el I Coloquio de Historia de la Educación colombiana, en Bogotá. Siendo el primero que se realizaba sobre esta temática en un país latinoamericano.

6 Resolución 2333 del 3 de Julio de 1998 del Ministerio de Educación Nacional de Colombia.

7 RUDECOLOMBIA, Red de Universidades Públicas Regionales de Colombia que se unieron para adelantar el primer doctorado de estas Instituciones en Ciencias de la Educación en el Área de Historia de la Educación Latinoamericana. Actualmente, componen la Red las Universidades de: Atlántico, Caldas, Cauca, Cartagena, Magdalena, Nariño, Tolima, Tecnológica de Pereira, Pedagógica y Tecnológica de Colombia y Quindío. 
nadora del Grupo de Investigación: Historia y prospectiva de la Universidad Latinoamericana. ${ }^{8}$ El Consejo editorial de la Revista quedó entonces conformado por los miembros de la Junta Directiva de SHELA: Pedro Alonso (España), Luis Celis Muñoz (Chile), Renate Marsiske (México), Jacobo Moquete (República Dominicana), Carmen Quintana (Paraguay) y María Cristina Vera de Flash (Argentina).

Está tradición del Consejo Editorial de la Revista se ha mantenido a través de los quince años de existencia de la revista, es decir, los miembros de la Junta Directiva de SHELA por derecho ocupan esta responsabilidad académica en la Revista. La Secretaría de este primer número la realizó el Dr. Carlos Londoño. Este cargo es rotativo y la Directora de la Revista invita a un especialista, según la temática, con la aprobación del Consejo Editorial.

La editora de la revista fue nombrada por la Junta Directiva de la Sociedad de Historia de la Educación Latinoamericana en la reunión que realizó la Junta en noviembre de 1997 en la ciudad de Tunja, Colombia, y se ha venido ratificando en el nombre de Diana Soto Arango en cada asamblea general de la Sociedad, su última designación fue el 26 de julio de 2013 en la ciudad de Barquisimeto, Venezuela, en la marco de la celebración de los 15 años de RHELA. Desde los inicios, la Revista cuenta con un Comité Científico, Comité Editorial y Consejo Asesor Internacional que garantizan la su calidad académica y científica. En el primer número estuvieron: Eve-Marie Fell (Francia), Pilar Gonzalbo (México), Celina Lértora (Argentina), Gabriel Restrepo (Colombia), Carlos Paladines (Ecuador) y Carlos Rincón (Alemania).

RHELA se caracteriza por la publicación de estudios monográficos de educadores latinoamericanos, aspecto que se ha mantenido desde la primera edición de la Revista. Este primer número se le dedicó al maestro Andrés Bello. En este apartado se destaca la colaboración del Doctor Javier Ocampo, que ha sido permanente durante estos años.

El primer número se dividió en cinco secciones: Artículos, Documentos, Informes de investigación, Reseñas de libros, Informes de la Sociedad. Las secciones se han mantenido, con pequeñas variaciones. En algunos números

8 Véase la historia de este grupo en "Boletín de Historia de la Educación Latinoamericana", Número 16 editado en el año 2012 con motivo de los 20 años del grupo de investigación, desde el año 1998 y hasta el 2012 acompañó en la coordinación del Grupo HISULA, el Dr. Carlos Rincón. A partir de esa fecha, colabora el Dr. José Rubéns Jardilino de la Universidad de Ouro Preto, Brasil. 
se ha agregado la Sección de Experiencias educativas. Los colaboradores de la Revista, en este primer número, fueron los miembros de la Sociedad de Historia de la Educación Latinoamericana. ${ }^{9}$ Se evaluaron 18 trabajos y se publicaron de estos seis artículos, un documento, un informe de investigación, que fue precisamente el proyecto de investigación "Historia de la universidad colombiana, 1774-1992"10 que sustentó la propuesta del Doctorado en Ciencias de la Educación de RUDECOLOMBIA, mediante convenio del 4 de junio de 1996. La Revista desde los inicios se ofreció como palestra para presentar avances de investigaciones de todas las personas que tienen como tema central historia de la educación en Latinoamericana.

Se retoma para este balance histórico lo señalado en la primera editorial, que definió la línea de investigación de la Revista y la comunidad académica a la que se dirigía, cuando se citó a John Weis:

...la historia es una ciencia útil. El pasado puede decirnos algo acerca del futuro. La sabiduría del historiador puede que radique en saber lo que ha ocurrido con anterioridad (...) nuestro conocimiento de lo que ha ocurrido previamente también puede convertirse fácilmente en el atisbo de lo que nos depara el futuro. Confiemos en que dicho conocimiento histórico sea sobre todo un medio para labrar nuestro destino. ${ }^{11}$

En definitiva, la Revista Historia de la Educación Latinoamericana, identificada con ISSN 0122-7238 para las ediciones en papel y con ISSN (versión online) 2256-5248, que se organizó y apareció editada por primera vez en mayo del año 1998, fue presentada ante la comunidad Latinoamericana durante la celebración del IV Congreso Iberoamericano de Historia de la Educación Latinoamericana en la ciudad de Santiago de Chile. Se cumplía de esta manera el objetivo señalado en 1992 de "dar a conocer los resultados de investigación de los miembros de la Sociedad y otros trabajos que se consideren relevantes para el avance de los estudios de nuestra Área."12

Una forma de difusión de la Revista, que se dio desde el origen de la publicación y se ha cristalizado como costumbre permanente, consiste en que

9 Esta Sociedad ha contado en su dirección, en calidad de Presidentes, con: Dra. Diana Soto Arango, de Colombia (septiembre 1994mayo1998); Dr. Luís Celis Muñoz, de Chile (mayo 1998-noviembre 2002); Dra. Maria Cristina Vera, de Argentina (noviembre 2002-octubre 2007); Dr. Jose Rubens Jardilino, Brasil (Octubre de 2007-agosto 2011); Dr. José Pascual Mora, Venezuela (agosto 2011).

10 Proyecto presentado por el grupo de investigación Historia y Prospectiva de la Universidad Latinoamericana (HISULA).

11 Moradielos, E. Título completo de la obra (1996).

12 Diana Soto Arango, (1998). 
los miembros del Doctorado en Ciencias de la Educación -el profesor y el estudiante-, en cada congreso o pasantía académica, llevan un paquete adicional en su maleta con la última edición de la Revista Historia de la Educación Latinoamericana. De esta manera, la publicación se da a conocer en el evento y se deja en las bibliotecas de la institución y de la ciudad. Igualmente, los miembros de la Sociedad de Historia de la Educación Latinoamericana llevan dos ejemplares: uno para la institución y el otro para su biblioteca personal.

Hay que decir que en octubre de 1997, SHELA, en su versión de Colombia como 'Red', ${ }^{13}$ organizó la Revista de Historia de la Educación Colombiana como órgano de expresión del proyecto "Historia y prospectiva de la universidad colombiana, 1774-1992" del Grupo de Investigación Historia y prospectiva de la Universidad Latinoamericana. El primer director de la Revista fue el Dr. Javier Ocampo López. Actualmente, la dirección de esta Revista está a cargo de la Dra. Gabriela Hernández, en la Universidad de Nariño.

En el contexto general latinoamericano, la Revista Historia de la Educación Latinoamericana fue la pionera en esta temática. Posteriormente, en el año 1998 aparecen las revistas de historia de la educación de Colombia y Chile. A partir del año 2001 inician las publicaciones sobre el tema de la historia de la educación de cada país en Argentina, Brasil y Venezuela. Sin lugar a dudas, el hecho que impulsó estos desarrollos de investigación y editoriales sobre la temática de la historia de la educación ha sido la Sociedad de Historia de la Educación Latinoamericana, que a su vez ha originado sociedades y redes sobre esta temática en los diferentes países de América Latina.

Hay que decir que, con antelación al año 1998, los investigadores de historia de la educación publicaban en las revistas de educación de sus respectivos países. Estas revistas tienen tradición desde el siglo XX y la más antigua se localiza en Chile, está desde finales del siglo XIX. En Colombia en el siglo XIX se creó la primera revista en educación, la Revista Escuela Normal, en el año 1870, y se editó hasta 1881 por parte del Ministerio de Instrucción

13 Esta Red se fue cristalizando a través de los Coloquios de Historia de la Educación Colombiana. En el año 1989 se organizó el I Coloquio en Bogotá, coordinado por Diana Soto Arango. El segundo, en la Universidad Pedagógica y Tecnológica de Colombia, en Tunja, en el año 1997, coordinado por Myriam Báez y Diana Soto Arango; el tercero, se realizó en la Universidad del Cauca, Popayán en el año 1999 coordinado por Elio Fabio Gutiérrez. El cuarto, en Bogotá, en el año 2005, coordinado por María Teresa Álvarez de la Universidad de Nariño. El quinto se organizó en Paipa en el año 2006, coordinado por María Teresa Álvarez, Jorge Tomás Uribe y Lina Parra dentro de los 10 años de RUDECOLOMBIA. El sexto se organizó, en agosto del 2008, en la Universidad Pedagógica y Tecnológica de Colombia, coordinado por Olga Acuña. 
Pública, luego se convirtió en Periódico Oficial de Instrucción Pública; La Revista Educación y Cultura se organizó en 1933, la Revista Educación de la Facultad de Educación de la Universidad Nacional de Colombia se edita de 1933 a 1935, posteriormente se reedita en julio de 1941, pero bajo la dirección y administración de la Escuela Normal Superior.

La Revista Maestro fue creada por el Ministerio de Educación Nacional en 1936 y se publicó hasta 1946; en 1978 aparece la Revista Educación Colombiana, adscrita al centro de investigaciones de la Universidad Pedagógica Nacional con 62 números editados a la fecha; en 1985 se edita la Revista Educación y Ciencia de la Facultad de Educación de la Universidad Pedagógica y Tecnológica de Colombia, con 14 números editados al 2012; en 1989 se publica la Revista Educación y Pedagogía de la Facultad de Educación de la Universidad de Antioquia, con 61 números editados. Para 1998, paralelo a la edición de RHELA, se publica la Revista Historia de la Educación Colombiana del Doctorado en Ciencias de la Educación de la Universidad de Nariño. ${ }^{14}$ Es relevante señalar el impacto de la Revista Interamericana de Educación, que aparece en 1912 y desaparece en 1951, esta publicación fue editada en Cali. En el año 2003 se organiza la Revista Internacional del Magisterio con 57 números editados al 2012. Finalmente para el año 2008 se edita la Revista Internacional de investigación en Educación de la Universidad Javeriana.

En el contexto Latinoamericano se destacan las revistas en educación más antiguas, como es el caso de las revistas chilenas. Para 1969 el Boletín de Educación de la Universidad Católica del Norte; La Revista Limes editada en 1988 por la Universidad Metropolitana de Ciencias de la Educación; en 1993 el Consejo Superior de Educación edita la Revista Calidad en la Educación, la Revista Boletín de Investigación Educacional; y la Revista Pensamiento Educativo de la Pontificia Universidad Católica de Chile surge en el año 1994.

Para el caso de Brasil se encuentra, en 1971, La Revista Cadernos de Pesquisa em Educação de La Fundação Carlos Chagas; en 1976 se publica la Revista Educação \& Realidade de la Universidade Federal do Rio Grande do Sul; en 1982 aparece la Revista Educação de la Universidade Federal de Santa Maria; en el año 1993 surge la Revista Educação: Teoria e Practica de la Universidade

14 Las cinco primeras ediciones de la Revista Historia de la Educación Colombiana las publicó el Doctorado en Ciencias de la Educación de la Universidad Pedagógica y Tecnológica de Colombia, a partir del número 6 la edita el Doctorado en Ciencias de la Educación de la Universidad de Nariño. 
Estadual Paulista Julio de Mesquita Filho; en 1997 se edita la Revista Historia da Educação de Asociação sul-Rio Grandense de Pesquisadores em Historia da Educação; y en 1999 en la Uninove se edita la Revista Eccos, la cual hace parte del convenio marco de cooperación editorial -entran también la Revista Historia de la Educación Latinoamericana y otras revistas- firmado en el 31 de agosto de 2011 en la ciudad de Manizales.

Igualmente, entre las revistas argentinas más antiguas se encuentran en 1994, la Revista Espacios en Blanco enditada de la Universidad Nacional de la Provincia de Buenos Aires, de igual forma, en el año 2005 se publica la Revista Historia de la Educación-Anuario adscrita a la Sociedad Argentina de Historia de la Educación.

En Costa Rica se identifican, para 1977 la Revista de Educación, y en el 2001 la Revista Actualidades Investigativas en Educación de la Universidad de Costa Rica. Por otra parte, en Cuba se edita la Revista Cubana de Educación Superior en el año 1981 del Centro de Estudios para el perfeccionamiento de la Educación Superior. En Perú se encuentra en 1990 la Revista Universidad Femenina del Sagrado Corazón; en 1997 la Revista Investigación Educativa de la Universidad Nacional Mayor de San Marcos.

Finalmente, está en Puerto Rico la Revista Cuadernos en investigación editada por el instituto libre de enseñanza, en 1987. En Venezuela se publica desde 1990 la Revista Ciencias de la Educación de la Universidad de Carabobo, y en 1995 Laurus: Revista de Educación de la Universidad Pedagógica Experimental Libertador y la Revista HUDERES, de la sociedad de Historia de la educación de Venezuela.

La característica común de todas las revistas de historia de la educación localizadas en los países de América Latina es la de ser el vehículo de difusión de los trabajos de las sociedades y / o Redes de esta temática en cada país. Por otra parte, hay revistas que están vinculadas a universidades que las apoyan en su financiación, y a su vez son el medio de publicación de las investigaciones de programas académicos. Por ejemplo, la Revista de Historia de la educación brasilera está localizada en la Facultad de Educación de la universidad de São Paulo.

Ahora bien, la única Revista de Historia de la Educación Latinoamericana es la nuestra, pionera en su género, que, como se ha indicado, reúne las caracterís- 
ticas de revistas de impacto internacional. El comité editorial ha establecido la prospectiva de publicación al año 2016 con el fin de dar a conocer a los investigadores las temáticas que se analizarán en los números siguientes. Esta estrategia ha visibilizado a RHELA como una publicación con calidad académica que da a los autores la posibilidad de preparar sus artículos inéditos y enviarlos al número donde se publican temas de su especialidad.

\section{Tabla 1. Prospectiva de publicación de RHELA al 2016}

\begin{tabular}{|c|c|c|}
\hline Número & Fecha de publicación & Tema \\
\hline 21 & 2 Semestre 2013 & $\begin{array}{c}\text { Políticas Publicas, reformas, } \\
\text { Movimientos estudiantiles }\end{array}$ \\
\hline 22 & 1 Semestre2014 & Género y Educación \\
\hline 23 & 2 Semestre 2014 & Masonería y educación \\
\hline 24 & 2 Semestre 2015 & Enseñanza Ilustrada \\
\hline 25 & 2 Semestre 2015 & Prensa y educación \\
\hline 26 & 2 Semestre 2016 & Educación ambiental \\
\hline
\end{tabular}

Fuente: Elaboración de las autoras

\section{Organización de la Revista}

La organización de la Revista se vincula con la Misión y Visión de la Sociedad de Historia de la Educación Latinoamericana, el Doctorado en Ciencias de la Educación de RUDECOLOMBIA y al grupo de investigación Historia y prospectiva de la Universidad Latinoamericana. En concreto, esta Revista presenta como Misión posicionar en el contexto internacional los resultados de investigación sobre Historia de la Educación Latinoamericana y colaborar en la consolidación de esta comunidad académica e investigativa, permitiendo vehicular los trabajos, al rescatar históricamente los valores éticos para la formación ciudadana y la construcción de la nación en el continente americano, en el compromiso de formar nuevos investigadores y editores de publicaciones científicas.

Por lo anterior, tiene como objetivo prioritario impulsar la investigación en la Historia de la Educación Latinoamericana; colaborar en la consolidación de la comunidad académica e investigativa de SHELA, al desarrollar el 
compromiso de ser el medio de difusión y consulta de los académicos y estudiantes en formación en historia, política, educación, que ubiquen sus investigaciones en la realidad educativa de Latinoamérica y promuevan los proyectos de rescatar los valores éticos, formación ciudadana y la construcción de la nación en el continente americano y a los orientadores de la política pública educativa de los países de Latinoamérica.

La Revista, hasta el número 9, se estructuró en cinco secciones. La primera, se dedicó a varios temas pero, a partir de la segunda, se ha destinado cada número a temas monográficos. Se finaliza la primera sección con un estudio biográfico del educador seleccionado para el respectivo número de la revista. Seguidamente, se ubica la segunda parte que corresponde a un documento relevante del educador señalado. El tercer apartado comprende artículos de reflexión que son avances de los proyectos de investigación. La cuarta, corresponde a reseñas de libros y revistas. La quinta, se dedica a reseñas de las actividades de eventos de la Sociedad de Historia de la Educación Latinoamericana.

A partir del número 10, la organización interna comprende cinco secciones: Artículos, Documentos, Reseña de libros y revistas, Reseñas de eventos e Información para autores.

La revista se caracteriza por los niveles en investigación y editorial de una publicación internacional. El proceso riguroso de selección se establece, desde la recepción de los artículos, en la verificación del cumplimiento de los requisitos editoriales mínimos de la Revista. Cada texto seleccionado es evaluado por dos pares internacionales especialistas en el tema al cual se dedica la edición, en ocasiones, y teniendo en cuenta el historial del artículo, se envía a un tercer par. Si el nuevo árbitro indica que se puede publicar, con las anotaciones se envía al autor y queda en la base de datos para analizar la posibilidad de publicación en un próximo número.

En la Revista se emplea la metodología de no dar un rechazo tácito al trabajo sino, por el contrario, colaborar en la formación de los investigadores, enviando los trabajos a los autores para su revisión. En la cuarta etapa del proceso editorial se analiza en conjunto con el Comité de publicaciones del Doctorado en Ciencias de la Educación de la Universidad Pedagógica y 
Tecnológica de Colombia y se toman las decisiones con el editor invitado en la parte final de las evaluaciones.

Finalmente, se envía el machote a revisión de estilo en español, inglés y portugués, y de esta manera se inicia el trabajo editorial del diseño y comprobación de la calidad de edición. La Editora, junto con el coordinador editorial, ${ }^{15}$ revisa todo el proceso. En el apoyo logístico se contó, en los inicios y hasta el año 2000, con Ana Ussa. ${ }^{16}$ En el año 2007 estuvo el joven investigador de HISULA Yules Alejandro Espinosa, y desde el 2008 y hasta la fecha está en el proceso editorial la Mg. Sandra Liliana Bernal Villate, joven investigadora de HISULA, quien realiza la tarea de asistencia editorial. A partir del 2012, en la edición número 18 ingresó en esta labor de acompañamiento de la visibilidad de la publicación la joven investigadora del grupo HISULA Lic. Mónica Valbuena Porras.

En el año 2006 se realizó un concurso Internacional para el Consejo Asesor 'Árbitros Internacionales', siendo seleccionados 27 currículos de 11 países, dentro de los 53 presentados. Estos países fueron Argentina Alemania, Brasil, Colombia, Ecuador, España, Honduras, México, Perú, Rusia y Venezuela.

El Comité Científico quedó representado por siete investigadores de cinco países. El nuevo Consejo Asesor y Científico inició funciones en la Revista número 8. Es importante destacar que con motivo de los diez años se realizó nuevamente el concurso para el Consejo Asesor 'Árbitros Internacionales'. Se presentaron 80 currículos y se seleccionaron 30. En esta ocasión quedaron investigadores de formación interdisciplinaria de los siguientes 22 países: Argentina, Alemania, Brasil, Bolivia, Colombia, Cuba, Chile, China, Ecuador, España, Estados Unidos, Honduras, Inglaterra, Japón, México, Perú, Puerto Rico, Portugal, Paraguay, República Dominicana, Rusia y Venezuela.

15 Han colaborado en la Coordinación Editorial: números 1 y 2, Dr. Carlos Londoño (Colombia); número 3, Dr. Justo Cuño Bonito (España); número 4, Dra. Laura López de la Cruz (España), número 5, Jesús Paniagua (España); Número 6, Dra. Myriam Báez Osorio (Colombia) y Dr. Alejandro Ávila (España); número 7, Dr. Jorge Tomás Uribe Ángel (Colombia); número 8, Prof. William Pacheco Vargas (Colombia); números 9, 10 y 11, Dr. Álvaro Acevedo Tarazona (Colombia); número 12, Dr. Antonio Elías de Pedro (México); número 13, Dr. José Rubens Lima Jardilino (Brasil); número 14, Dr. Justo Cuño Bonito (España); número 15, Dra. María Isabel Lafuente (España); número 16, Nina Hasewagua (país); número 17, Thérèse Hamel (Canadá), José Pascual Mora (país); número 18, Alba Nidia Triana (país) y Oscar López (país).

16 Del año 2001 a 2005 a la Editora le correspondió, en solitario, realizar la labor de editor asistente en el envío y recepción de los artículos con los autores y los evaluadores, incluyendo el ingreso de la información del primer formulario de Indexación de la Revista en Publindex. 
Para el año 2012 se llevó a cabo el concurso para comité científico y comité asesor internacional, se recibieron 70 currículos, que fueron objeto de evaluación, según los criterios de indexación establecidos por Publindex en Colombia, de esta forma los comités quedaron conformados de la siguiente manera: Comité científico integrado por 6 investigadores de 6 países iberoamericanos: Brasil, España, Francia, México, Portugal y Venezuela; el Comité editorial integrado por 6 investigadores de 5 países e integrantes de SHELA, entre estos se destacan Brasil, Colombia, Cuba, México Perú y Venezuela, y finalmente el Consejo asesor internacional, integrado por 17 investigadores de 8 países: Argentina, Alemania, Brasil, Cuba, Chile, España, México y Perú. Finalmente la Revista establece, a partir del 2013, una evaluación semestral de los comités. La característica común de todos los miembros del comité científico y editorial es que tengan título de doctor y publicaciones en revista indexada.

\section{Visibilidad de los Educadores Latinoamericanos}

Se ha indicado que desde los inicios de la Revista se propuso estudiar el pensamiento de los educadores latinoamericanos. El objetivo, respecto a los educadores, se ha centrado en analizar y poner de manifiesto las ideas y las prácticas educativas que se desplegaron en el continente con el impacto de los pensamientos e ideas, que en materia de educación, se desarrollaron a través del tiempo y las incidencias que han tenido en el desarrollo y transformación cultural-educativa del continente americano. Se ha tomado al educador como un actor social en unos espacios de poder local, nacional e internacional. ${ }^{17}$

Se conecta a estos personajes con las tendencias del pensamiento científicoeducativo avanzado de la época. Los estudios se orientan a la historia de las ideas, la cultura y la educación. Se identificará desde la historia socio-política a los educadores que hayan impactado, con la práctica y/o escritos, en la transformación educativa de sus países y en otras regiones de América y como esas ideas ayudaron a constituir en cada periodo las concepciones de nación en el continente. ${ }^{18}$

\footnotetext{
17 Proyecto Educadores Latinoamericanos que con la colección se inscribe en el Grupo de Investigación Historia y Prospectiva de la Universidad Latinoamericana.

18 Este objetivo se cristalizó en el proyecto de Educadores en América Latina y el Caribe. Siglos XVI a XXI. (nov. 2007). El proyecto es coordinado por Diana Soto Arango, Jesús Paniagua y José Rubens Jardilino.
} 
Desde esta perspectiva, se ha tomado cada educador incluyendo siempre un documento inédito o poco conocido de gran relevancia que exprese el aporte del educador. El primer educador que inició la Revista fue el venezolano Maestro Don Andrés Bello (1781-1865). Se le ha catalogado a Andrés Bello como uno de los ideólogos del "nacionalismo cultural de Hispanoamérica", con gran influencia en las generaciones del siglo XIX. El documento seleccionado fue el "Discurso de Don Andrés Bello en la instalación de la Universidad Chilena, el 17 de septiembre de 1843". ${ }^{19}$

El segundo educador correspondió al argentino Domingo Faustino Sarmiento (1811-1888), denominado "el maestro de escuela que llegó a la presidencia" y, como lo sintetiza el Dr. Ocampo, "autodidacta que tuvo como pasión la educación, la primera empresa del Estado". El documento que se publicó fue sobre: "La educación de la mujer. Discurso inaugural en el Colegio de Santa Rosa de San Juan, 9 de julio de 1839". ${ }^{20}$

El volumen tres se dedicó al peruano ilustrado Pablo de Olavide (1725-1803), se destaca su labor de reformador de la educación universitaria hispalense, quien concebía la universidad como la institución que forma el personal del estado. El Documento editado corresponde a la "Reforma del Plan de estudios, del 12 de febrero de 1768". ${ }^{21}$ Este Plan fue pionero en la metrópoli española y marco influencia en los criollos ilustrados de América colonial.

La Revista, en su cuarta edición, presentó a la chilena Gabriela Mistral (1889-1957), a quien se le ha caracterizado por su función de maestra de escuela rural, diplomática y Premio Nobel de Literatura. Resulta interesante encontrarle el documento "La escuela, la maestra rural" y "la oración de la maestra" ${ }^{22}$ por los valores reflejados en estos escritos de Lucila de María del Perpetuo Socorro Godoy Alcayata, su verdadero nombre.

El volumen cinco presentó al impulsor de la Escuela Nueva en Latinoamérica. Al colombiano Agustín Nieto Caballero (1889-1975). Se publicó el

19 Andrés Bello, "Discurso de instalación de la Universidad Chilena el 17 de septiembre de 1843" Revista Historia de la Educación Latinoamericana No. 1 (1998): 151.

20 Domingo Sarmiento 9 de julio de 1893, documento publicado en la Revista Historia de la Educación Latinoamericana No. 2 (2000): 139.

21 Pablo Olavide, "Reforma del Plan de estudios, del 12 de febrero de 1768" Revista Historia de la Educación Latinoamericana No. 3 (2001):231 - 237.

22 Gabriela, Mistral, “La escuela, la maestra rural' y 'la oración de la maestra” Revista Historia de la Educación Latinoamericana No. 4 (2002): 247 - 249 
documento Principios de Escuela Nueva ${ }^{23}$ que aplicó en la experiencia educativa que dirigió del Colegio Gimnasio Moderno en Bogotá desde el año 1918.

Al político-educador de Puerto Rico, Eugenio María de Hostos (1839-1903) se le dedicó el volumen seis. Hostos, desde el positivismo y defendiendo los valores éticos morales, le apostó a la formación de maestros y organizó la Escuela Normal de Santo Domingo. Además, planteó reformas educativas para la educación secundaria desde la rectoría del Liceo Chillán, en Chile. El documento que se seleccionó y publicó en este volumen fue sobre "La educación de la mujer"24 que lo expuso en Santo Domingo en el año 1881.

El volumen siete le correspondió al impulsor de reformas educativas, el mexicano José Vasconcelos (1882-1959). Su idea de "hacer de la escuela una casa del pueblo y del maestro un líder de la comunidad" marcó derroteros en Latinoamérica en la primera mitad del siglo XX. La Revista publicó en este número tres documentos de Vasconcelos: De Robinson a Odisea. 1955, Discurso en la Universidad, 1920, y Discurso pronunciado en el día del maestro. $1923 .{ }^{25}$

En la edición número ocho se destaca la labor del brasileño Darcy Ribeiro (1922-1997) quien marcó el derrotero del análisis sobre las reformas universitarias de Latinoamérica. El documento que se publicó versó sobre El experimento de la universidad de Brasilia. 1964. ${ }^{26}$

El maestro del libertador, el venezolano Simón Rodríguez (1769-1853), fue el invitado en el volumen nueve y se publicó el documento "Extracto sucinto de mi obra sobre educación republicana, presenta el pensamiento de este educador venezolano, quien con sus ideas de la ilustración influyo en la formación del Libertados Simón Bolívar"27.

Paulo Freire (1921-1997) fue el educador brasileño analizado en la edición número diez, uno de los grandes pensadores contemporáneos de la educación

23 Agustín Nieto Caballero "Principios de la Escuela Nueva”, Revista Historia de la Educación Latinoamericana No. 5, (2003): 139.

24 Eugenio María, Hostos "La educación de la mujer" Revista Historia de la Educación Latinoamericana No. 6, (2004):251 - 260

25 José Vasconcelos, documentos publicados en la Revista Historia de la Educación Latinoamericana No. 7, (2005):161-183.

26 Darcy Ribeiro, "El experimento de la universidad de Brasilia, 1964" Revista Historia de la Educación Latinoamericana No. 8, (2006): 161.

27 Simón Rodríguez, "Extracto sucinto de mi obra sobre la educación republicana” Revista Historia de la Educación Latinoamericana No. 9, (2007): 105. 
Latinoamericana, el Dr. Javier Ocampo se centró en su obra más conocida, Pedagogía del oprimido, el gran educador de la educación popular acompañó la Revista en su número diez, se publicó en la sección de documentos un texto de Freire, casi desconocido que se titula O profesor universitario como educador..$^{28}$

En la edición número once se abordó la temática sobre movimientos estudiantes en América Latina, dedicando este volumen a Germán Arciniega, maestro colombiano (1900-1999), como el gestor de Federación de Estudiantes de Colombia, quien inicio el movimiento estudiantil en la década de los veinte del siglo XX, el documento que acompaña esta edición fue el Manifiesto de Córdoba de 1918, el Manifiesto de Mayo Francés y el Programa Mínimo de 1971, importantes en el estudio y análisis del Movimiento Estudiantil en América Latina.

El colombiano Orlando Flas Borda (1925-2008) fue el protagonista del número doce, se destacan sus ideas educativas y la metodología científica de la Investigación Acción Participación, en esta edición se publicaron once artículos donde se analiza la calidad de la educación en Iberoamérica, de igual forma se publica como documento el Honoris Causa al Dr. Orlando Flas Borda.

El volumen número trece dedicado a la educadora brasileña Nísia Floresta Brasileira (1810-1885) y la influencia de su práctica educativa en el siglo XIX, a su vez, se dan a conocer los resultados del proyecto de investigación Educadores en América Latina y el Caribe siglos XVI al XXI, en esta edición se estudian los aportes académicos de educadores casi desconocidos fuera de su frontera nacional. En la sección de documentos se edita la declaración de los derechos de la mujer y de la ciudadana Onlympe de Gouces 1789 y las principales obras inéditas de Nísia Floresta. ${ }^{29}$

José Félix de Restrepo, colombiano (1760-1832), fue el protagonista del volumen número catorce, se destaca al educador como el maestro de la generación de independencia en Colombia, quien desde Popayán, Antioquia y Bogotá formó numerosos políticos de la primera república Granadina y la

28 Paulo Freire, “O profesor universitario como educador" Revista Historia de la Educación Latinoamericana No. 10 (2008):139.

29 Revista Historia de la Educación Latinoamericana No. 13 (2008). 
Gran Colombia. ${ }^{30}$ Como documento se publica el texto titulado El Estado de la Instrucción y Educación Pública en la Nueva Granada 1815-1820.

El número quince presenta al educador mexicano Justo Sierra (1848-1912), desde sus ideas positivistas, fue considerado como uno de los grandes educadores y humanistas de México y fundador de la Universidad Nacional Autónoma de México, se publicó el documento inauguración de la Universidad Nacional de México el 22 de septiembre de $1910 .{ }^{31}$

El volumen dieciséis convoca al educador ecuatoriano Francisco Febres Cordero (1854-1910), y se abordó la temática de análisis de textos escolares. "Se resalta este educador como autor de textos escolares con mayor influencia en Hispanoamérica desde finales del siglo XIX y la primera mitad del siglo $X X^{\prime \prime}$; el documento que acompaña la edición corresponde a los textos publicados por Febres Cordero.

La edición diecisiete visibiliza a la educadora argentina Juana Paula Manso (1819-1875) El tema sobre el cual versan los trabajos se refiere a los educadores y a las problemáticas en torno al ejercicio de la función docente e investigadora, como documento se presenta la cronología de la educadora y sus principales publicaciones. ${ }^{32}$

El número dieciocho se dedica a la educadora argentina Rosario Vera Peñaloza (1873-1950), la edición se dedica a la maestra rural, el documento que acompaña la publicación es el libro copiador de puño y letra de la educadora en el Colegio de Buenos Aires Escuela Normal Roque Sáenz Peña.

Para el 2012 la edición número 19 presenta a la comunidad académica una publicación en torno a las Nuevas Tecnologías y visibiliza en su carátula al educador venezolano José Silvio Pomenta (1943-2009) como uno de los especialistas a nivel internacional en el campo de las TICS, se publica como documento una semblanza al educador elaborada por Sandra Mendoza Verde y la hija del educador, Patricia Silvio, este documento se acompaña

30 Javier Ocampo López, "El maestro José Félix de Restrepo, el educador de la Generación de Independencia en Colombia”, Revista Historia de la Educación Latinoamericana, Vol. 14 (2010): 19-160.

31 Justo Sierra, "Inauguración de la Universidad Nacional de México el 22 de septiembre de 1910", Revista Historia de la Educación Latinoamericana No. 15, (2010): 325.

32 Cronología elaborada por la Dra. Gabriela Hernández directora del Doctorado en Educación RUDECOLOMBIA de la Universidad de Nariño. 
de una relación de recursos en Internet, destacados para la academia y la investigación.

En celebración de los 15 años de publicación de RHELA, se edita en el 2013 la edición número 20 dedicada a la educadora Cubana Dulce María Borrero (1883-1945) en este artículo la autora hace un análisis del su perfil intelectual, contextualizado en sus contribuciones de esta maestra a la educación republicana de la primera mitad del siglo XX en Cuba, el documento que acompaña la edición es un apartado del texto titulado la La ornamentación de la escuela de Dulce María Borrero. ${ }^{33}$

En definitiva, veinte educadores(as), han sido motivo de nuestro estudio y el Doctor Javier Ocampo siempre ha estado presto a colaborar con el análisis biográfico de la mayoría de ellos y en la selección de los documentos. Estos educadores provienen de 10 países latinoamericanos: de Argentina (3), Brasil (3), Cuba (1), Colombia (4), Chile (1), Ecuador (1), México (2), Perú (1), Puerto Rico (1) y Venezuela (3). De estos pertenecen 4 al siglo XVIII, 10 al siglo XIX y 6 al siglo XX.

De esta manera, los veinte educadores(as) han marcado las directrices temáticas de la revista y han tocado los tópicos sobre universidad, escuelas normales, educación popular, escuelas, escuela nueva, currículo, movimientos estudiantiles, textos escolares, educadores, maestras rurales, TICS, los lenguajes revistas en Iberoamérica. Finalmente, se señala que como propósito para los próximos números se dará visibilidad a educadoras y educadores con impacto regional, poco conocidos en los contextos internacionales.

\section{Internacionalización y Aspecto Físico de RHELA}

La Revista es de carácter internacional por la representatividad de los investigadores, en los escritos y la representatividad de los evaluadores provenientes de diferentes países de tres continentes, ${ }^{34}$ y por la visibilidad y catalogación en nueve índices que dan cuenta de la calidad académica de los artículos y la calidad editorial.

33 Dulce María Borrero. “La ornamentación de la escuela”, Revista de Instrucción Pública, Año II, No. 3, marzo, (1926): 238-244.

34 Los Continentes de: América, Europa y Asia. 
El Comité Científico y el Consejo Asesor de Árbitros Internacionales se caracterizan porque todos sus miembros tienen título de doctor y publican en el año, mínimo un artículo en revista indexada y / o capítulo de libro.

La Revista se localiza en papel en las Bibliotecas nacionales de la mayoría de los países de América Latina; en las universidades de los miembros de la Sociedad de Historia de la Educación Latinoamericana, en varios Institutos de Estudios Latinoamericanos de España, Francia, Inglaterra y Estados Unidos. Se tiene canje con más de sesenta instituciones. Se difunde en todos los eventos de Sociedad de Historia de la Educación Latinoamericana y de RUDECOLOMBIA.

En cuanto al aspecto físico, se utiliza en la edición en papel un tamaño de $17 \mathrm{x}$ $24 \mathrm{~cm}$, papel propalibros de 90 gramos a una tinta, carátula en propalcote de 240 gramos brillante. En cuanto al envió de artículos deben ser inéditos, con una extensión máxima de 20 cuartillas, incluidos cuadros, gráficos, notas, imágenes relacionadas con el tema, apéndices y bibliografía. Se deben enviar en Microsoft Word, tamaño carta, a doble espacio, tipo de letra Times New Roman, tamaño 12, con márgenes superior e inferior de $3 \mathrm{~cm}$ y márgenes derecho e izquierdo de $2,5 \mathrm{~cm}$. Los trabajos se componen de: introducción, desarrollo de contenidos sobre la temática solicitada, conclusiones, fuentes y referencias. En papel se editan los textos completos en el idioma español y portugués y cuentan con el respectivo resumen en inglés. En las páginas www.rhela.rudecolombia.edu.co y http://virtual.uptc.edu.co/revistas / index.php/historia_educacion_latinoamerica se publica la versión completa de los textos en inglés, a partir del número 18. La versión electrónica se identifica con el ISSN 2256-5248 desde al año 2012.

La Revista ha tenido una preocupación constante por estar dentro de los parámetros internacionales de calidad en la investigación y en la calidad editorial, utilizando criterios de comparabilidad. Por tal motivo, las normas editoriales se han tomado de la revisión de las Revistas de circulación y calidad científica internacional. ${ }^{35}$ Las normas de citación de RHELA son adaptación de The Chicago Manual of Style, en su versión "Humanities Style". El diseño de la carátula se sometió a concurso en el quinto número. El concurso lo ganó el Dr. Antonio de Pedro. La revista número 8, del año 2006, presentó un diseño novedoso, pero dentro de la auto-evaluación de cada número

35 Se ha recibido asesoría editorial de Pedro Sánchez de Ediciones Doce Calles de España y el Dr. Miguel Ángel Puig-Samper Mulero, director de Publicaciones del Consejo Superior de Investigaciones Científicas de España (CSIC) y de la Dra. Consuelo Naranjo. 
se localizó que este diseño correspondía más a una revista de divulgación que a una de carácter científico. Por este motivo se ha regresado al diseño clásico, donde solo se utiliza diagramación con inclusión de gráficos según la temática del artículo.

En cuanto al proceso de indexación la Revista Historia de la Educación Latinoamericana hace parte de los siguientes catálogos, bases bibliográficas, índices y sistemas de indexación.

\section{Indexación y Homologación}

Publindex Índice Nacional de Publicaciones Seriadas Científicas y Tecnológicas de Colombianas - Colciencias, 2001. Actualmente categoría B.

\section{Bases Bibliográficas con Comité de Selección}

Redalyc - Red de Revistas Científicas de América Latina y el Caribe España y Portugal <http:/ / redalyc.uaemex.mx/>

Fuente Académica - EBSCO, 2009 <http:/ / search.ebscohost.com>

\section{Catálogos}

Latindex - Sistema Regional de Información en Línea para Revistas Científicas de América Latina, el Caribe, España y Portugal <www.latindex.unam.mx>

Dialnet - Fundación Dialnet <http://dialnet.unirioja.es/servlet/revista?tipo_ busqueda $=$ CODIGO\&clave_revista $=10778>$

CLASE - Citas Latinoamericanas en Ciencias Sociales y Humanidades. http:/ / clase.unam.mx/

$D O A J<$ http: / / www.doaj.org/>

IISUE <www.iisue.unam.mx/iresie/>

REBIUN <http: / rebiun.absysnet.com/ cgi-bin/ abnetop/X16883 /

ID1072319373?ACC $=161>$

\section{Portales web y repositorios}

$<$ http:/ / virtual.uptc.edu.co/revistas/index.php/historia_educacion_latinoamerica> 
(versión español e inglés) Página web de la revista en el portal de la UPTC $<$ www.rhela.rudecolombia.edu.co $>$ (versión española e inglés) Página Web de la Revista

$<$ http:/ / www.grupodeinvestigacionhisula.com> Página web del grupo de investigacion HISULA

$<$ www.grupodeinvestigacionilac.com $>$ Página web del grupo de investigacion ILAC

$<$ www.publicaciones.rudecolombia.edu.co/publicaciones $>$

http: / / www.shela-hedu.org Página web de la Sociedad de Historia de la Educación Latinoamericana

\section{Redes Sociales}

Facebook: rhela@rudecolombia.edu.co

Twitter: @RHELAUPTC

Se destaca que en Latindex se localizan revistas de 39 países de Iberoamérica. En los cinco primeros lugares se registra a: Brasil, con 4724 revistas; España, 3500; Argentina, 3283; México, 2414 y Chile con 1881. Colombia solo tiene indexadas 687 revistas en Latindex. En Educación y Ciencias Humanas se registran en Latindex 53 revistas. De estas 11 corresponden a Colombia y dentro de estas se localiza la Revista Historia de la Educación Latinoamericana. Para la visibilidad e internacionalización de la Revista, se ha optado, igualmente, por el mecanismo de presentarla en las Ferias Internacionales del Libro. En el 2007 se inició en abril en Bogotá, Colombia y en noviembre en Guadalajara, México. Año tras año se presenta en la Feria internacional del libro en Bogotá, Colombia y en los congresos internacionales de americanistas, FIEALC, ISCHE, Americanistas, SHELA.

\section{Dificultades, logros y prospectiva}

Un proyecto editorial de una revista se logra cuando se convierte en un proyecto colectivo. Los inicios, como en todo proyecto, son difíciles hasta obtener la credibilidad en su medio académico. Pero quizá, el gran enemigo de las publicaciones periódicas es su financiación. Se debe señalar que esta Revista se ha financiado ya que no se le paga a los evaluadores y en cada número se inicia un proceso de búsqueda de recursos. Hasta la fecha la ha financiado la Sociedad de Historia de la Educación Latinoamericana de 
forma permanente. Las otras ayudas han provenido, según el número, de la Facultad de Educación y Dirección de Investigaciones de la Universidad Pedagógica y Tecnológica de Colombia, Universidad Pablo de Olavide, Universidad de Sevilla, España, Universidad de León España y RUDECOLOMBIA de forma permanente desde el año 2011 junto con el Doctorado Ciencias de la Educación de la Universidad Pedagógica y Tecnológica de Colombia.

Los años difíciles ya pasaron. La Revista tiene una posición, impacto y visibilidad internacional. Es referenciada en investigaciones, tesis doctorales y revistas. La voluntad de los investigadores es manifiesta al enviar sus trabajos y los asesores que los evalúan. Sin embargo, aún queda un largo camino por recorrer. La financiación es un problema no resuelto, el equipo técnico logístico está en proceso de mejoramiento y el cumplimiento de las fechas por parte de los evaluadores es una asignatura pendiente.

En todo caso, estas problemáticas se resolverán porque se tiene garantizada la comunidad académica de investigadores que cada día incrementa las investigaciones y por lo tanto la Revista realiza la prospectiva dentro de la visión de un plan estratégico del $2010^{36}$ al 2018. ${ }^{37}$ Se espera que en dos años este medio de publicación se posicione en el contexto nacional e internacional por el excelente nivel académico manifestado a través de la inclusión en los medios de Indexación más reconocidos. En los próximos diez años será interlocutor válido, por su consulta de parte de investigadores, asesores en las políticas públicas educativas de cada país de Latinoamérica, por colaborar en la consolidación de la comunidad académica e investigativa de la Sociedad de Historia de la Educación Latinoamericana y por su compromiso en la formación de historiadores y educadores en el contexto euro americano, desde una dimensión ética y comprometida en los proyectos de formación ciudadana y construcción de la nación en Latinoamérica.

\section{CONCLUSIÓN}

La Revista Historia de la Educación Latinoamericana aparece en 1998 con el ISSN 0122-7238, en el 2012 se registra con ISSN electrónico 2256-5248. En el año 2001 ingresa en Publindex a través de Colciencias desde el Doctorado en Ciencias de la Educación de la Universidad Pedagógica y Tecnológica de

36 Fecha de conmemoración del bicentenario del inicio de las independencias americanas.

37 Fecha cuando se cumple 20 años de la Revista Historia de la Educación Latinoamericana. 
Colombia. En el transcurso de los quince años, se han editado 20 números y se localiza en 9 índices. Posee página Web con todos los artículos en español y, a partir del número 7, también se localizan en inglés, a partir del número 18, correspondiente al primer semestre de 2012, se editan en la versión online en español y en inglés. En estos 20 números se han editado 238 artículos con una participación de 217 investigadores, provenientes de 19 países. La Revista, además de tener la circulación en Internet, se localiza en las Bibliotecas nacionales de la mayoría de los países latinoamericanos y en un gran número de instituciones universitarias y de investigación. Se ha presentado en varias Ferias Internacionales del libro, entre las que se destaca en el 2007: La de abril en Bogotá, Colombia, y la de noviembre en Guadalajara, México. En el mes de abril del 2008 en Bogotá, Colombia y en Madrid, España. Se distribuye por medio electrónico y en papel por medio de empresas editoriales.

Hay que decir que esta revista ha impulsado un proyecto científicoacadémico donde se unen, bajo un mismo objetivo, una sociedad académica y un programa de formación doctoral. Desde los inicios, ha marcado una política de impulso a la investigación apoyándose en los grupos de Sociedad de Historia de la Educación Latinoamericana y del Doctorado en Ciencias de la Educación. Esta Revista es pionera en su género en Latinoamérica. La Revista Historia de la Educación Latinoamericana, se ha distinguido de otras publicaciones por su marcada internacionalización referenciada a partir de los colaboradores y su difusión.

Es relevante resaltar la labor de visibilidad a los educadores latinoamericanos que, según la temática de la Revista, se publica la biografía de estos, recatando y publicando un documento poco conocido. En definitiva, se considera que con esta Revista se inició una nueva etapa para los intelectuales e investigadores de la Historia de la Educación de finales del siglo XX en América Latina. La Sociedad y el Doctorado han pasado por diferentes períodos históricos de rupturas y dificultades. Sin embargo, la Revista, con el problema central de la financinaciación, se ha mantenido gracias a ese grupo de "soñadores de proyectos utópicos" como nos catalogaron en el año 1992.

En resumen puede indicarse que el aporte de la Revista ha radicado en impulsar la investigación y dar un medio de publicación de alto nivel académico internacional que se ha puesto al servicio de esos dos grandes proyectos, referenciados, de asociación académica modelos para el continente ameri- 
cano. Por lo anterior, se puede afirmar que la Revista en sus quince años, personalizada en el Comité Editorial versus Junta Directiva de SHELA, se ha caracterizado por convocar y liderar la investigación histórico-educativa en América Latina y el Caribe.

\section{FUENTES}

Bello, Andrés, "Discurso de Don Andrés Bello en la instalación de la Universidad Chilena, el 17 de septiembre de 1843", Revista Historia de la Educación Latinoamericana, No, 1. (1998).

Hostos, Eugenio María, "La educación de la mujer. 1881", Revista Historia de la Educación Latinoamericana, No, 6. (2004): 251.

Moradielos, E. El oficio del historiador (Madrid: Siglo XXI de España, 1996).

Nieto Caballero, Agustín, "Principios de Escuela Nueva”, Revista Historia de la Educación Latinoamericana, No. 5 (2003): 139 - 143.

Olavide, Pablo, "Reforma del Plan de estudios, del 12 de febrero de 1768", Revista Historia de la Educación Latinoamericana, No. 3 (2001): 175 - 230.

RUDECOLOMBIA, UPTC, Revista Historia de la Educación Latinoamericana, No. 1, (Tunja: 1998).

RUDECOLOMBIA, UPTC, Revista Historia de la Educación Latinoamericana, No. 2, (Tunja: 2000).

RUDECOLOMBIA, UPTC, Revista Historia de la Educación Latinoamericana, No. 3, (Tunja: 2001).

RUDECOLOMBIA, UPTC, Revista Historia de la Educación Latinoamericana, No. 4, (Tunja: 2002).

RUDECOLOMBIA, UPTC, Revista Historia de la Educación Latinoamericana, No. 5, (Tunja: 2003).

RUDECOLOMBIA, UPTC, Revista Historia de la Educación Latinoamericana, No. 6, (Tunja: 2004).

RUDECOLOMBIA, UPTC, Revista Historia de la Educación Latinoamericana, No. 7, (Tunja: 2005).

RUDECOLOMBIA, UPTC, Revista Historia de la Educación Latinoamericana, No. 8, (Tunja: 2006).

RUDECOLOMBIA, UPTC, Revista Historia de la Educación Latinoamericana, No. 9, (Tunja: 2007).

RUDECOLOMBIA, UPTC, Revista Historia de la Educación Latinoamericana, No. 10, (Tunja: 2008).

RUDECOLOMBIA, UPTC, Revista Historia de la Educación Latinoamericana, No. 11, (Tunja: 2008).

RUDECOLOMBIA, UPTC, Revista Historia de la Educación Latinoamericana, No. 12, (Tunja: 2009).

RUDECOLOMBIA, UPTC, Revista Historia de la Educación Latinoamericana, No. 13, (Tunja: 2009).

RUDECOLOMBIA, UPTC, Revista Historia de la Educación Latinoamericana, No. 14, (Tunja: 2010).

RUDECOLOMBIA, UPTC, Revista Historia de la Educación Latinoamericana, No. 15, (Tunja: 2010).

RUDECOLOMBIA, UPTC, Revista Historia de la Educación Latinoamericana, No. 16, (Tunja: 2011).

RUDECOLOMBIA, UPTC, Revista Historia de la Educación Latinoamericana, No. 17, (Tunja: 2011).

RUDECOLOMBIA, UPTC, Revista Historia de la Educación Latinoamericana, No. 18, (Tunja: 2012).

RUDECOLOMBIA, UPTC, Revista Historia de la Educación Latinoamericana, No. 19, (Tunja: 2012).

Sarmiento, Domingo, “La educación de la mujer. Discurso inaugural en el Colegio de Santa 
Rosa de San Juan. 9 de julio de 1839", Revista Historia de la Educación Latinoamericana, No. $2(2000): 139$.

Soto Arango, Diana. "Editorial", Revista Historia de la Educación Latinoamericana, No. 1. (1998).

Vasconcelos, José, "De Robinson a Odisea. 1955", "Discurso en la Universidad. 1920", y "Discurso pronunciado en el día del maestro. 1923", Revista Historia de la Educación Latinoamericana, No. 7, (2005): 177.

Soto Arango, Diana Elvira y Bernal Villate, Sandra Liliana. "Revista Historia de la Educación Latinoamericana. Líder en la investigación histórico-educativa 1998-2013". Revista Historia de la Educación Latinoamericana. Vol. 15 No, 20, (2013): $45-70$. 